\title{
Information Hiding Method Based on Block DWT Sub-Band Feature Encoding
}

\author{
Qiudong SUN, Wenxin MA, Wenying YAN, Hong DAI
}

School of Electronic and Electrical Engineering, Shanghai Second Polytechnic University, Shanghai, China.

Email: \{qdsun,wxma, wyyan, daihong\}@ee.sspu.cn

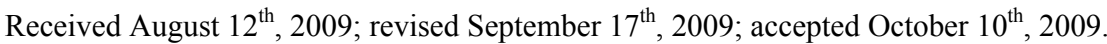

\begin{abstract}
For realizing of long text information hiding and covert communication, a binary watermark sequence was obtained firstly from a text file and encoded by a redundant encoding method. Then, two neighboring blocks were selected at each time from the Hilbert scanning sequence of carrier image blocks, and transformed by 1-level discrete wavelet transformation (DWT). And then the double block based JNDs (just noticeable difference) were calculated with a visual model. According to the different codes of each two watermark bits, the average values of two corresponding detail sub-bands were modified by using one of JNDs to hide information into carrier image. The experimental results show that the hidden information is invisible to human eyes, and the algorithm is robust to some common image processing operations. The conclusion is that the algorithm is effective and practical.
\end{abstract}

Keywords: Sub-Band Feature Encoding, Redundant Encoding, Visual Model, Discrete Wavelet Transformation, Information Hiding

\section{Introduction}

With the development of information technology, people have paid more and more attention to the information security. Information hiding in a digital image has become the focus of the information security research. For an effective information hiding scheme, three basic requirements should be satisfied: transparency, robustness and security. The former two are in conflict with each other. To dissolve this conflict availably, we can consider using the masking characteristic of human visual system (HVS) [1]. Duo to its good time-frequency localization function is similar to the visual masking of HVS, the DWT has been used widely in the field of information hiding [2]. A good hiding technique should also extract the hidden information from stego-image blindly.

In recent years, many algorithms based on HVS and DWT had been proposed for information hiding [1-6]. And some [1-3] of them also had implemented the blind extraction of hidden information. But those algorithms are mostly armed at binary iconic watermark. So they are unsuitable for hiding the text information and covert communication. The reference [5] proposed a robust encryption technique for text information. Though the transparency, robustness and security of that algorithm were all good, the embedded capacity was restricted due to only one bit watermark can be hidden in two blocks, whose sizes were settled as $8 \times 8$.
Use for reference [5] in watermark embedding, we propose an adaptive information hiding method based on average value relation of corresponding DWT sub-bands of two neighboring blocks with double JND thresholds and adjustable block size. As mentioned previously, in order to adjust the input image for transparent watermarks, we employ a visual model $[2,5]$ to calculate the different double block based JND thresholds for determining the intensity of watermarking at the different location of image. We also give a redundant encoding method for robustness.

This paper is organized as follows. In Section 2, we give the JND threshold calculation equation for controlling the embedding intensity. Section 3 presents the information hiding algorithm and its extraction in detail. Section 4 examines the performance of proposed algorithm, and shows that the proposed scheme yields more effective and better performance, both in terms of transparency and robustness through simulation. Section 5 gives the conclusion of this paper.

\section{JND Threshold Calculation}

\subsection{Visual Model}

Under the background gray $f$, the human eyes relative sensitivity to gray change $\gamma(f)=\Delta f l f$, which is a non-linear function of $f$, can be approximated by the equation as follows [5]: 


$$
\gamma(f)=\frac{\Delta f}{f}=0.02\left[\mathrm{e}^{\frac{128}{f}-1}+\mathrm{e}^{\frac{1}{2256-f)} \frac{1}{128}}\right]
$$

where e is the base of natural logarithm. In experiment, we can use the gray mean of $K \times K$ image block $\boldsymbol{B}_{u v}$ located at $(u, v)$ as the background gray $f$, i.e. $f=$ mean $\left(\boldsymbol{B}_{u v}\right)$.

\subsection{JND Calculation}

To ensure the watermark has good transparency and robustness, we can use JND to adjust the intensity of watermark-embedding [2,4,5]. The image block $\boldsymbol{B}_{u v}$ is DWT-transformed into an approximate image and three detail sub-band images $\boldsymbol{D}_{u v}^{s}(s \in \mathrm{HH}, \mathrm{HL}, \mathrm{LH}$, represent the three detail sub-bands of diagonal, horizontal and vertical directions respectively). The JNDs of three detail sub-bands are represented as follows:

$$
\boldsymbol{J}_{u v}^{s}=\boldsymbol{T}_{u v} \boldsymbol{F}_{s}
$$

where $\boldsymbol{T}_{u v}$ is the normalized value of $\boldsymbol{T}_{u v}^{\prime}=f \gamma(f) \boldsymbol{E}_{u v}$ at the range $[a, b]$, while $\boldsymbol{E}_{u v}$ is the normalized entropy of $\boldsymbol{B}_{u v}$. When $s \in \mathrm{HH}, \boldsymbol{F}_{s}$ equals $\sqrt{2}$, otherwise it is 1 [4].

\section{Adaptive Information Hiding Scheme}

\subsection{Watermark Embedding}

Let $\boldsymbol{W}$ represents a watermark sequence, $\boldsymbol{B}_{u v 1}$ and $\boldsymbol{B}_{u v 2}$ are two neighboring image blocks and their DWT-transformed detail sub-bands are $\boldsymbol{D}_{u v 1}^{s}$ and $\boldsymbol{D}_{u v 2}^{s}$ (simply marked by $\boldsymbol{D}_{1}$ and $\boldsymbol{D}_{2}$, or by a universal symbol $\boldsymbol{D}_{t}, t \in\{1$, $2\}$ ). Now we can define the admissible distortion factor of sub-band coefficients of DWT as follows:

$$
\lambda_{t}=\frac{\left|\boldsymbol{D}_{\mathrm{t}}\right|+\delta}{\operatorname{mean}\left(\boldsymbol{D}_{\mathrm{t}} \mid\right)+\delta} \quad t \in\{1,2\}
$$

where $\delta$ is a positive number, which is an effect factor of absolute values of detail sub-band coefficients to embedding intensity. When the block size is $2 \times 2$, whatever the value of $\delta$ is, the Equation (3) is constant and can be simplified to $\lambda_{t}=1$.

We assume that $\mu \delta$ is the mean value of JNDs of two neighboring blocks. It is represented by equation as follows:

$$
\mu=\frac{1}{2}\left(\boldsymbol{J}_{u v 1}^{s}+\boldsymbol{J}_{u v 2}^{s}\right)
$$

If the crytic normalized range of $[a, b]$ in Equation (2) is set by two different un-overlapped ascend ranges $\left[a_{0}, b_{0}\right]$ and $\left[a_{1}, b_{1}\right]$, such as $[1,2]$ and $[6,7]$, then we can get two different $\mu$ from Equations (2) and (4). They can be represented by $\mu_{0}$ and $\mu_{1}$, or by a universal symbol $\mu_{r}$, $r \in\{0,1\}$.

We also assume that $\Delta d$ is the corresponding DWT detail sub-band coefficients difference of two neighbor- ing blocks at same direction, and $\varepsilon$ is the adjustment intensity matrix of detail sub-bands coefficients. They are represented by equations as follows respectively:

$$
\begin{aligned}
& \Delta d=\operatorname{Sign}\left(\boldsymbol{W}_{k}\right)\left[\operatorname{mean}\left(\boldsymbol{D}_{2}\right)-\operatorname{mean}\left(\boldsymbol{D}_{1}\right)\right] \\
& \boldsymbol{\varepsilon}_{t}=\frac{1}{2} \operatorname{Sign}\left(\boldsymbol{W}_{k}+t\right) \lambda_{t}\left(\mu_{\boldsymbol{W}_{k+1}}-\Delta d\right)
\end{aligned}
$$

where $\operatorname{Sign}(\cdot)$ is a sign function, it is defined as follows:

$$
\operatorname{Sign}(x)= \begin{cases}1 & x \in \text { even } \\ -1 & x \in \text { odd }\end{cases}
$$

$\boldsymbol{W}_{k}$ is the $k$-th element of binary watermark sequence $\boldsymbol{W}$.

If the DWT detail sub-band features of two neighboring blocks after embedded should satisfy the relationship with the consecutive two bits $\boldsymbol{W}_{k}$ and $\boldsymbol{W}_{k+1}$ of watermark sequence as shown in Table 1 . We can prove that the watermark embedding rule is as follows:

$$
\boldsymbol{D}_{t}^{\prime}= \begin{cases}\boldsymbol{D}_{t}+\boldsymbol{\varepsilon}_{t} & \text { if }\left(\boldsymbol{W}_{k+1}=1 \text { and } \Delta d<\mu\right) \text { or }\left(\boldsymbol{W}_{k+1}=0\right) \\ \boldsymbol{D}_{t} & \text { otherwise }\end{cases}
$$

From the embedding rule, we know that each couple corresponding detail sub-bands of two neighboring blocks can be embedded 2 watermark bits. And the two neighboring blocks have three couple corresponding detail sub-bands. So, if the size of carrier image is $M \times N$, the information hiding capacity of this algorithm can reach to the value of $(3 M N) /\left(K^{2}\right)$ bits. It is double than that of [5]. For example, if the size of carrier image is $512 \times 512$ and the block size is $2 \times 2$, then the full information hiding capacity is 196608 bits or 24576 bytes. It is large enough to hide information. If applied to hide short text information into a carrier image, this method can bring an enough redundancy to ensure its robustness.

\subsection{Watermark Extraction}

Being the same with watermark embedding, we should select two neighboring blocks $\boldsymbol{B}_{u v 1}^{\prime}$ and $\boldsymbol{B}_{u v 2}^{\prime}$ each time from Hilbert scanning sequence of stego-image blocks, and a couple of their DWT detail sub-bands $\hat{\boldsymbol{D}}_{1}$ and $\hat{\boldsymbol{D}}_{2}$. And set $t h=\left(b_{0}+a_{1}\right) / 2$. Then, we can prove that the watermark extraction rule is as follows:

Table 1. The relationship between watermark codes and the DWT detail sub-band features of two neighboring blocks

\begin{tabular}{cc}
\hline $\boldsymbol{W}_{\boldsymbol{k}}$ and $\boldsymbol{W}_{\boldsymbol{k}+\mathbf{1}}$ & $\begin{array}{l}\text { The size relationship of corresponding } \\
\text { DWT detail sub-bands }\end{array}$ \\
\hline 00 & $\operatorname{mean}\left(\mathbf{D}_{2}^{\prime}\right)-\operatorname{mean}\left(\mathbf{D}_{1}^{\prime}\right)=\mu_{0}$ \\
01 & $\operatorname{mean}\left(\mathbf{D}_{2}^{\prime}\right)-\operatorname{mean}\left(\mathbf{D}_{1}^{\prime}\right) \geq \mu_{1}$ \\
10 & $\operatorname{mean}\left(\mathbf{D}_{2}^{\prime}\right)-\operatorname{mean}\left(\mathbf{D}_{1}^{\prime}\right)=-\mu_{0}$ \\
11 & $\operatorname{mean}\left(\mathbf{D}_{2}^{\prime}\right)-\operatorname{mean}\left(\mathbf{D}_{1}^{\prime}\right) \leq-\mu_{1}$ \\
\hline
\end{tabular}




$$
\begin{gathered}
\hat{\boldsymbol{W}}_{k}= \begin{cases}0, & \text { if } \operatorname{mean}\left(\hat{\boldsymbol{D}}_{2}\right) \geq \operatorname{mean}\left(\hat{\boldsymbol{D}}_{1}\right) \\
1, & \text { else }\end{cases} \\
\hat{\boldsymbol{W}}_{k+1}= \begin{cases}0, & \text { if }\left[\operatorname{Sign}\left(\boldsymbol{W}_{k}\right)\left(\operatorname{mean}\left(\hat{\boldsymbol{D}}_{2}\right)-\operatorname{mean}\left(\hat{\boldsymbol{D}}_{1}\right)\right)\right]<t h \\
1, & \text { else }\end{cases}
\end{gathered}
$$

where $\hat{\boldsymbol{W}}_{k}$ is the $k$-th element of watermark sequence $\hat{\boldsymbol{W}}$, which is extracted from stego-image blindly. From Equation (9), we know that the anti-interference ability of this algorithm lies on the interval value between $b_{0}$ and $a_{1}$. The larger interval value is, and the better anti-interference ability is.

\subsection{Information Hiding Algorithm}

Step 1: Read a text file and convert it into a bit stream $\boldsymbol{W}$, which is called the original watermark.

Step 2: In order to enable that the length of original watermark $\boldsymbol{W}$ is just equal to 3 times of total blocks number of carrier image, some zeros can be appended to the end of it.

Step 3: For improving the robustness of watermarking, the redundancy of embedded watermarks should be ensured. So the original watermark $\boldsymbol{W}$ should be extended periodically as follows:

$$
\boldsymbol{W}_{\mathrm{ex}}^{m}=\boldsymbol{W}_{l} \quad m=n \cdot L+l ; n=0,1, \cdots, C r-1 ; l=0,1, \cdots, L-1
$$

where $\boldsymbol{W}_{\text {ex }}$ is the extended watermark, $\boldsymbol{W}_{\mathrm{ex}}^{m}$ represents its $\mathrm{m}$-th element, $\mathrm{Cr}$ is the extended factor.

Step 4: In order to improve the security of watermarking, $\boldsymbol{W}_{\text {ex }}$ should be scrambled randomly.

Step 5: In order to keep the relativity of two neighboring image blocks, we can scan the original carrier image by Hilbert scanning to obtain a Hilbert scanning sequence.

Step 6: Select two neighboring image blocks $\boldsymbol{B}_{u v 1}$ and $\boldsymbol{B}_{u v 2}$ each time from the Hilbert scanning sequence, and embed the watermark according to the method as mentioned in Section 3 until all DWT detail sub-bands of all blocks have been processed.

Step 7: After applied the inverse DWT for all watermark embedded blocks, we can get a stego-image $\boldsymbol{I}^{\prime}$.

\subsection{Information Recovering Algorithm}

Step 1: Scan the stego-image $\boldsymbol{I}$ by Hilbert scanning with the same order as that in information hiding.

Step 2: Select two neighboring image blocks $\boldsymbol{B}^{\prime}{ }_{u v 1}$ and $\boldsymbol{B}^{\prime}{ }_{u v 2}$ each time from the Hilbert scanning sequence, and extract the watermark according to the method as mentioned in Section 3 until all DWT detail sub-bands of all blocks have been processed.

Step 3: After that, we can get a watermark sequence $\hat{\boldsymbol{W}}_{\text {ex }}$, which involves the $C r$ copies of original watermark.
Step 4: If there was a scrambling when watermark was embedded, here we should do unscrambling to $\hat{\boldsymbol{W}}_{\text {ex }}$.

Step 5: The final watermark can be obtained from $\hat{\boldsymbol{W}}_{\text {ex }}$ as follows:

$$
\hat{\boldsymbol{W}}_{l}= \begin{cases}1, & \text { if } \sum_{n=0}^{C r-1} \hat{\boldsymbol{W}}_{\mathrm{ex}}^{n \cdot L+l} \geq \frac{C r}{2} \\ 0, & \text { else }\end{cases}
$$

Step 6: The binary watermark sequence $\hat{W}$ should be converted back into a text file.

\section{Experimental Results}

The peak signal-to-noise ratio (PSNR) is employed to evaluate the quality of stego-image, meanwhile the bit error rate (BER) is employed to evaluate the quality of recovered secret information.

In the experiment, the proposed algorithm was evaluated on the gray image "Lena" $(512 \times 512 \times 8)$. The block size $K$ can be 8,4 or 2 . In order to ensure the algorithm's anti-interference ability is good, $\left[a_{0}, b_{0}\right]$ should be set smaller and $\left[a_{1}, b_{1}\right]$ should be set bigger. So we set $\left[a_{0}, b_{0}\right]=[1,2]$. Figure 1 is the relationship between PSNR of stego-image and the effect factor $\delta$ at different block sizes. Figure 2 is the relationship between BER of the recovered secret bits and the effect factor $\delta$. Figure 3 is the relationship between PSNR and the JND normalized range $\left[a_{1}, b_{1}\right]$ at full capacity embedding.

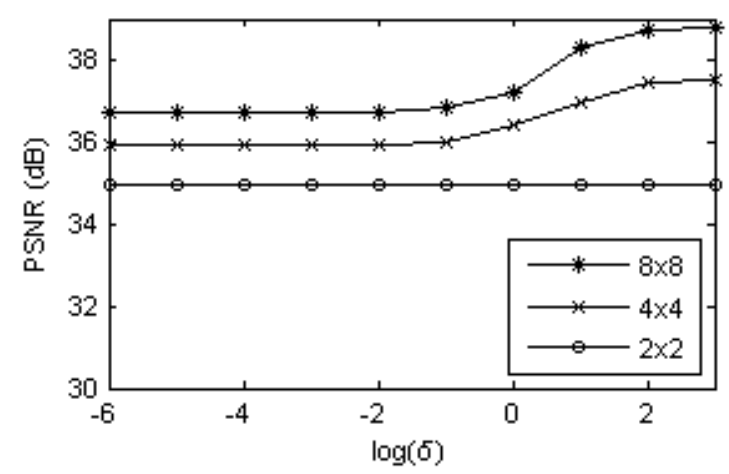

Figure 1. Relationship between PSNR and $\delta$

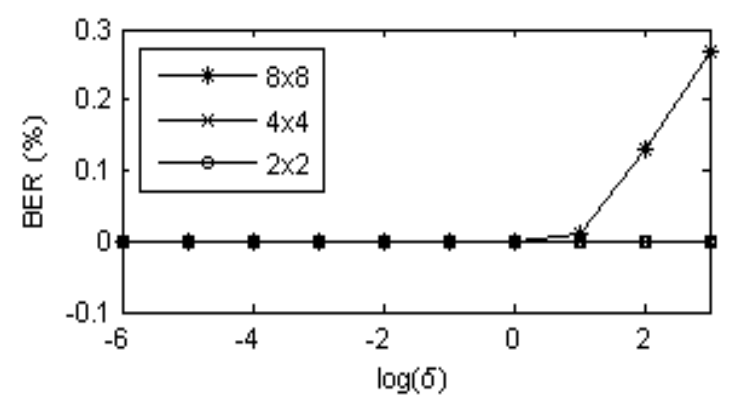

Figure 2. Relationship between BER and $\delta$ 


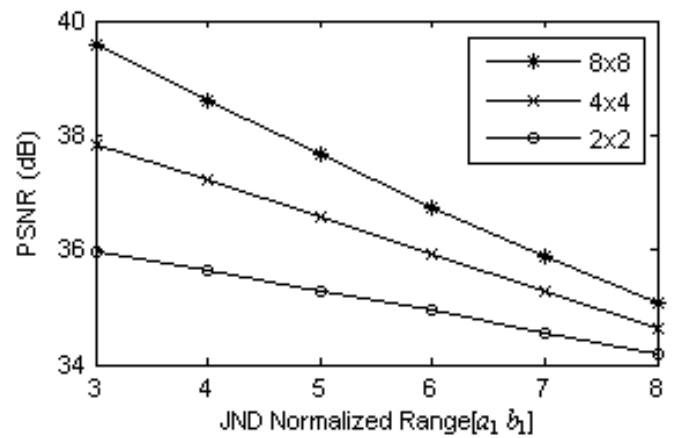

Figure 3. Relationship between PSNR and $a_{1}\left(\left[a_{0}, b_{0}\right]=[1,2]\right.$, $\left.b_{1}=a_{1}+1\right)$

As shown in Figure 1, the PSNRs of stego-image are constant when $K=2$, but when $K>2$, the bigger $\delta$ is, the higher PSNRs are. But as known in Section 3, the bigger $\delta$ will bring down on the embedding intensity, it leads that the performance of recovering algorithm will be worse, especially when $K=8$, the BERs will be higher. As shown in Figure 2, we know that BERs are always zeros when $\delta \leq 1$, and whatever the $K$ is. So we set the $\delta=1$ in the following experiments. As shown in Figure 3, the PSNRs are almost in inverse proportion to $\left[a_{1}, b_{1}\right]$. The smaller $\left[a_{1}, b_{1}\right]$ is, the higher PSNRs are, and the better imperceptibility of hidden information is. So that an appropriate $\left[a_{1}, b_{1}\right]$ is better. Here, we set $\left[a_{1}, b_{1}\right]=[6,7]$. As shown in Table 2, the algorithm's full hiding capacities in various block sizes and their performances are good enough to hide information.

Figure 4(a) is the original images of "Lena". Figure 4(b) and 4(c) are the stego-image hidden with 768 bytes text when $K=8$ and the difference images between the original image and stego-image on the condition of magnifying 30 times. Figure 4(d) and 4(e) are the results when $K=4$. Figure 4(f) and 4(g) are the results when $K=2$. As shown in Table 2 and Figure 4, considering the visual perception in the algorithm, the hidden information in "Lena" gray image is invisible though the PSNR is a little low. The algorithm can fully recover the hidden information from the stego-image.

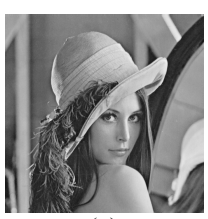

(a)

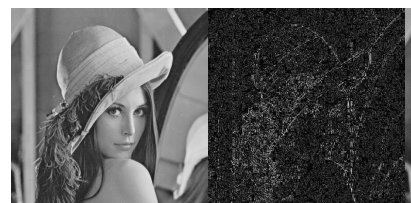

(d)

(e)

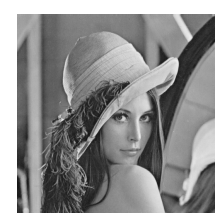

(b)

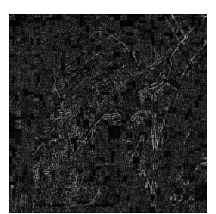

(c)

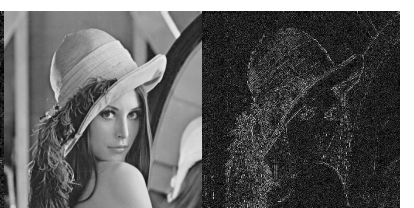

(g)

Figure 4. The information hidden results of "Lena"

Table 2. The full capacities in differenct block sizes and their PSNRs and BERs

\begin{tabular}{lccc}
\hline \multicolumn{1}{c}{$\begin{array}{c}\text { Capacity and Per- } \\
\text { formances }\end{array}$} & \multicolumn{3}{c}{ Block size } \\
\cline { 2 - 4 } & $8 \times 8(K=8)$ & $4 \times 4(K=4)$ & $2 \times 2(K=2)$ \\
\hline Hiding capacity (bit) & 12288 & 49152 & 196608 \\
Hiding capacity (byte) & 1536 & 6144 & 24576 \\
PSNR (dB) & 37.21 & 36.44 & 34.95 \\
BER (\%) & 0 & 0 & 0 \\
\hline
\end{tabular}

Table 3. The zero BER attack defense tests of short text information hiding

\begin{tabular}{lc}
\hline \multicolumn{1}{c}{ Attack items } & Performance under zero BER \\
\hline Cropping in central region & $258 \times 258$ \\
Brightness enhancement & $69 \%$ \\
Contrast enhancement & $33 \%$ \\
\hline
\end{tabular}

In the experiment, we also did some attacking tests to the algorithm on the condition of $K=4$ and 768 bytes text with 8 redundant copies. As shown in Table 3, we found that the algorithm was robust to central region cropping, brightness enhancement and contrast enhancement.

\section{Conclusions}

This paper presented a new scheme of information hiding in gray images based on DWT for long text information hiding or covert communication. In our approach, the comparability of corresponding DWT detail sub-bands of two neighboring image blocks was considered, and in order to improve the transparency of information hiding, the visual model was also used for calculating the double block based JNDs to determine the embedding intensity at different locations of image. The block DWT sub-band feature encoding technique increased the embedding capacity double than that of [5]. The adjustable block size gave facilities for various applications. If you request the algorithm to have a better transparency, you should select bigger block size. Or if you request that it has a larger embedding capacity, you should select smaller block size. In addition, in order to improve the algorithm's robustness and ability of defense some general image processing attacks, such as cropping, brightness enhancement and contrast enhancement, the redundant encoding was given for increasing the embedded copies of watermark. The experiment results demonstrate that the proposed algorithm yields the acceptable performance for transparency and robustness, and also increases the embedding capacity for information hiding.

\section{Acknowledgements}

This research project was supported by the Technological Innovation Foundation of Shanghai Municipal Education Commission under Grant No. 09YZ456 and the Key 
Disciplines of Shanghai Municipal Education Commission under Grant No. J51801.

\section{REFERENCES}

[1] M. Barni, F. Bartolini, and A. Piva, "Improved waveletbased watermarking through pixel-wise masking," IEEE Transactions on Image Processing, Vol. 10, No. 5, pp. 783-791, 2001.

[2] H. F. Yang and X. W. Chen, "A robust image-adaptive public watermarking technique in wavelet domain," Journal of Software, Vol. 14, No. 9, pp. 1652-1660, 2003.

[3] J. G. Cao, J. E. Fowler, and N. H. Younan, “An imageadaptive watermark based on a redundant wavelet trans- form," In: Pitas I, ed. Proceedings of the IEEE International Conference on Image Processing, Thessaloniki, pp. 277-280, 2001.

[4] Z. M. Wang, Y. J. Zhang, and J. H. Wu, "A wavelet domain watermarking technique based on human visual system," Journal of Nanchang University (Natural Science), Vol. 29, No. 4, pp. 400-403, 2005.

[5] Q. D. Sun, W. X. Ma, W. Y. Yan, and H. Dai, "Text encryption technique based on robust image watermarking," Journal of Image and Graphics, Vol. 13, No. 10, pp. 1942-1946, 2008

[6] L. Y. Wu and F. Yang, "An improved digital watermarking algorithm based on DWT," Control and Automation, Vol. 23, No. 6(3), pp. 46-47, 59, 2007. 